\title{
XMM and INTEGRAL monitoring of Mrk 509: The origin of X-ray emission in AGN
}

\section{Stéphane Paltani ${ }^{* 1}$, P.-O. Petrucci ${ }^{2} ;$ M. Mehdipour ${ }^{3}$, G. Ponti ${ }^{4} ;$ R. Detmers ${ }^{5}$, J.} Malzac $^{6}$, P. Lubinski ${ }^{7}$, J. Kaastra ${ }^{5,8}$, S. Bianchi ${ }^{9}$, M. Cappi ${ }^{10}$, K. Steenbrugge ${ }^{11,12}$, G.A. Kriss ${ }^{13}$, N. Arav ${ }^{14}$, A. Blustin ${ }^{3,15}$, G. Branduardi-Raymont ${ }^{3}$, E. Costantini ${ }^{5}$, M. Dadina $^{10}$, C. de Vries ${ }^{5}$

${ }^{1}$ ISDC Data Centre for Astrophysics, Observatory of the University of Geneva, Switzerland

${ }^{2}$ UJF-Grenoble 1/CNRS-INSU, Institut de Planétologie et d'Astrophysique de Grenoble, France

${ }^{3}$ Mullard Space Science Laboratory, University College London, United Kingdom

${ }^{4}$ School of Physics and Astronomy, University of Southampton, UK

${ }^{5}$ SRON Netherlands Institute for Space Research, Utrecht, The Netherlands

${ }^{6}$ Centre d'Étude Spatiale des Rayonnements, Université de Toulouse/CNRS, Toulouse, France

${ }^{7}$ Centrum Astronomiczne im. M. Kopernika, Toruń, Poland

${ }^{8}$ Sterrenkundig Instituut, Universiteit Utrecht, The Netherlands

${ }^{9}$ Dipartimento di Fisica, Università degli Studi Roma Tre, Italy

${ }^{10}$ INAF-IASF Bologna, Italy

${ }^{11}$ Instituto de Astronomía, Universidad Católica del Norte, Antofagasta, Chile

${ }^{12}$ University of Oxford, Department of Physics, United Kingdom

${ }^{13}$ Space Telescope Science Institute, Baltimore, USA

${ }^{14}$ Department of Physics, Virginia Tech, Blacksburg, USA

${ }^{15}$ Institute of Astronomy, University of Cambridge, United Kingdom

E-mail: Stephane.Paltani@unige.ch

In October-November 2009, our collaboration performed an unprecedented joint XMMINTEGRAL campaign consisting of 10 4-day separated observations of the bright Seyfert 1 galaxy Mrk 509, totaling $600 \mathrm{ks}$ for XMM and 1.2 Ms for INTEGRAL, complemented by HST, Chandra, Swift/XRT and ground-based optical observations. Among the numerous science goals, we present here the study of the broad-band emission, from the UV up to the hard X-rays (0.003$250 \mathrm{keV}$ ), which we attempt to model with physically motivated models. The variability pattern of the UV and X-ray emission points to a Comptonization origin of the soft-excess in a warm, very optically thick plasma. The absence of detectable high-energy cut-off $\left(E_{\mathrm{C}}>200 \mathrm{keV}\right)$ and the high UV-to-X ratio point to the existence of a very hot $\left(\mathrm{k} T_{\mathrm{C}} \sim 500 \mathrm{keV}\right)$, optically-thin $(\tau \sim 0.025)$ corona. The corona parameters are found to be consistent with those expected from a passive disk in radiative equilibrium with the corona in a slab geometry.

8th INTEGRAL Workshop "The Restless Gamma-ray Universe”- Integral2010,

September 27-30, 2010

Dublin Ireland

\footnotetext{
*Speaker.

${ }^{\dagger}$ POP acknowledges financial support from CNES

${ }^{\ddagger}$ GP acknowledges Marie Curie European Fellowship support under contract no. FP7-PEOPLE-2009-IEF-254279
} 


\section{Introduction}

Hard X-ray emission from Seyfert galaxies is most often described as a simple exponential cutoff power-law [2], with a photon index typically around $\Gamma=1.9$. This mathematical description is however unsatisfactory if one wants to understand the intrinsic functioning of active galactic nuclei. Physical modeling of this component generally assumes that its origin lies in Comptonization of soft photons from the accretion disk. The presence of a cut-off energy points however to the existence of a thermal population of hot electrons in a medium surrounding the accretion disk [21], akin to the Solar corona. Unfortunately, the necessary capabilities to observe this emission in hard $\mathrm{X}$-rays with high sensitivity are still largely missing, and thermal-Comptonization models have been applied only to a small number of bright Seyfert galaxies [10, 16, 17, 22]. These studies point to the existence of rather hot $(\mathrm{k} T \sim 100 \mathrm{keV})$, moderately optically-thick plasma $(\tau \sim 1)$. Plasma geometry and heating mechanisms are however still poorly constrained.

The soft-excess, an excess of emission observed below $2 \mathrm{keV}$ compared to the extrapolation of a power-law fitted to the $2-10 \mathrm{keV}$ emission, is another common signature of Seyfert 1 galaxies. Its origin is hotly debated. Thermal Comptonization of accretion disk emission has been proposed as a possible explanation for the soft-excess [10]. In particular, the correlation of the soft-excess strength with that of the blue-bump is a strong argument in favor of this mechanism [20]. However, the constancy of the plasma temperature across very different black-hole masses makes this mechanism rather improbable [7], although this result could be affected by some observational bias. Models based on ionized reflection in the immediate vicinity of the black hole [1, 4] or ionized absorption in high-velocity winds $[6,7]$ successfully reproduce the shape of the X-ray emission, including reverberation delays [5].

A very intensive observation campaign on the bright Seyfert 1 galaxy Mrk 509 offers a new opportunity to test the physical mechanisms at play in this object. In this paper, we study the broad-band continuum emission of Mrk 509 from the ultraviolet up to the hard X-rays. We attempt to model the blue-bump emission, the soft-excess and the hard X-ray emission with physically motivated models. We show here the preliminary results of this work and some first attempts at interpreting them.

\section{The Mrk 509 campaign}

Mrk 509 has been observed 10 times over the period October-November 2009, once every four days quasi-simultaneously (i.e., with large overlaps) with XMM-Newton and INTEGRAL. The average exposure time per observation was $60 \mathrm{ks}$ for XMM-Newton and $120 \mathrm{ks}$ for INTEGRAL. Complementary observations with HST, Chandra, Swift/XRT and ground-based optical telescopes have been obtained before and after the XMM-INTEGRAL campaign over a span of 3 months. The first goal of the XMM-Newton campaign is the study of the warm-absorber physical properties through reverberation [8]. However, the quasi-simultaneous INTEGRAL observations also allow us to study the broad-band emission properties and variability from the ultraviolet up to the hard X-rays.

We focus here exclusively on the XMM-Newton/OM (photometry only), XMM-Newton/pn and INTEGRAL/ISGRI data. XMM/OM and XMM/pn data have been reduced with SAS v9.0; 

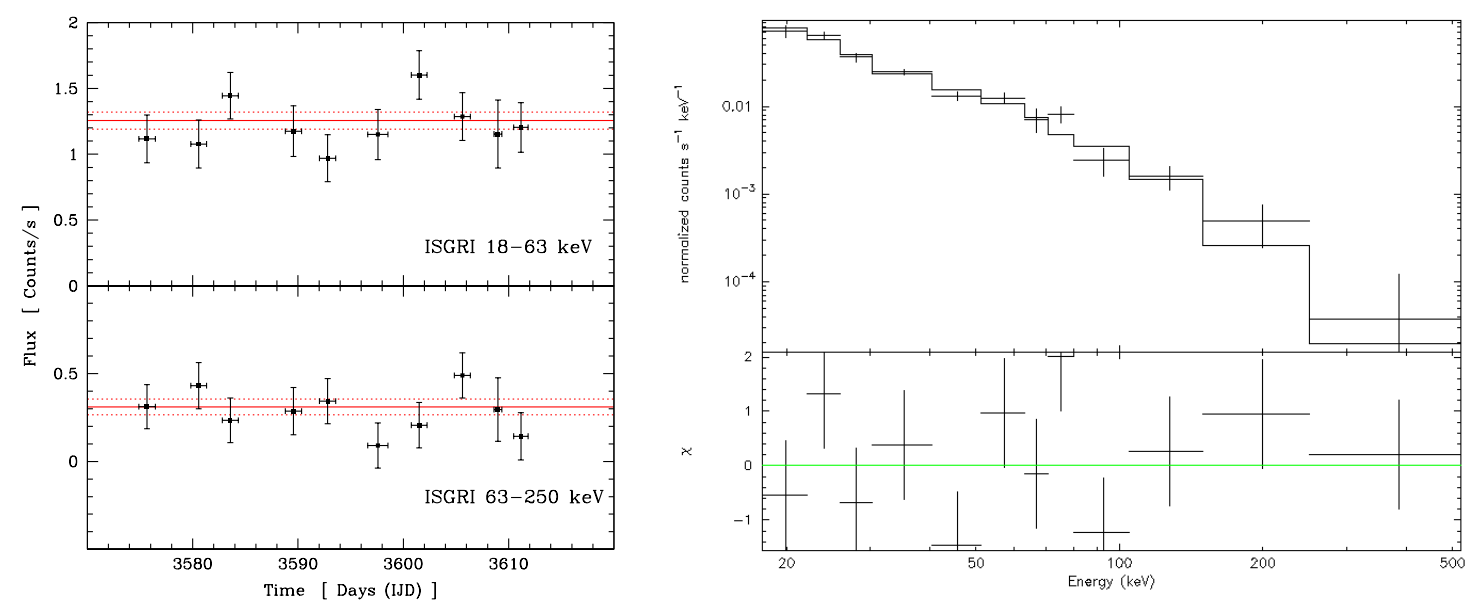

Figure 1: INTEGRAL/ISGRI observations. Left: ISGRI light curves in two energy bands. The red lines show the average flux and its $\pm 1 \sigma$ uncertainty. Right: Average ISGRI spectrum. The spectrum is modeled with a simple power-law with a best-fit photon index $\Gamma=2.02 \pm 0.17$

details about the processing are presented elsewhere $[12,18]$. In addition, the properties of the accretion disk component in the ultraviolet (namely, its peak temperature) have been determined from HST/COS and archival FUSE observations [12]. INTEGRAL/ISGRI data have been reduced with OSA 8, with the addition of the ghost cleaning improvements provided in OSA 9. The presence of a SPI annealing cutting some $40 \%$ of the SPI observations during this campaign unfortunately prevented us from reaching adequate sensitivity in the SPI energy bands.

\section{Results}

\subsection{INTEGRAL observations}

Figure 1 shows the INTEGRAL/ISGRI light curves in two energy bands (18-63 keV and 63$250 \mathrm{keV}$ ) and the average spectrum fitted with a simple power-law. The total on-source exposure time is $1.02 \mathrm{Ms}$. Mrk 509 is detected at $3.5 \sigma$ in the $100-150 \mathrm{keV}$ energy bin. The $18-63 \mathrm{keV}$ light curve is formally compatible with a constant flux, as is the $63-250 \mathrm{keV}$ light curve. We fit the average spectrum with a simple power-law model. The fit is acceptable $\left(\chi^{2}=12.3\right.$ for 12 degrees of freedom), with a photon index $\Gamma=2.02 \pm 0.17$, without any evidence for the presence of a cutoff. Constraining the intrinsic photon index to remain larger than $\Gamma=1.8$, the average spectrum even allows us to exclude the presence of a cut-off at energies below $E_{\mathrm{C}} \simeq 200 \mathrm{keV}$.

\subsection{Flux correlations}

Figure 2 (left) shows the correlations between the different light curves extracted from the $\mathrm{XMM} / \mathrm{OM}, \mathrm{XMM} / \mathrm{pn}$ and INTEGRAL/ISGRI instruments. One XMM/OM light curve covers the UV emission from 3 to $6 \mathrm{eV}$, six XMM/pn light curves cover the soft and medium X-rays from 0.2 to $12 \mathrm{keV}$ and one INTEGRAL/ISGRI light curves cover the hard X-rays from 18 to $63 \mathrm{keV}$. The $63-250 \mathrm{keV}$ has been left out because of its very poor $\mathrm{S} / \mathrm{N}$. The correlation pattern is very regular, with all light curves being strongly correlated with their immediate neighbors, the 

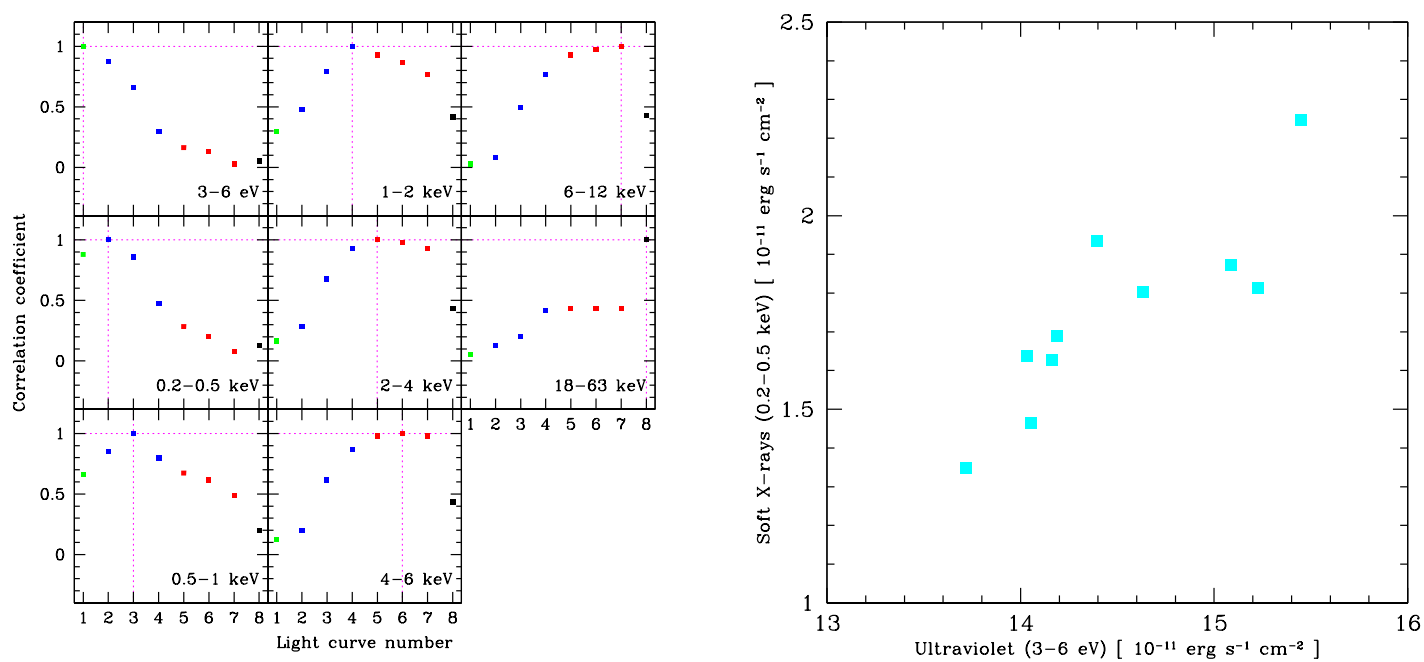

Figure 2: Left: Flux-flux correlation coefficients in the different bands for the $10 \mathrm{XMM}+\mathrm{INTEGRAL}$ observations. In each panel, light curves are numbered: (1) XMM/OM UV light curve in the 3-6 eV band; $\mathrm{XMM} / \mathrm{pn}$ soft X-ray bands: (2) $0.2-0.5 \mathrm{keV}$, (3) $0.5-1 \mathrm{keV}$, (4) $1-2 \mathrm{keV}$; XMM/pn medium X-ray bands: (5) 2-4 keV, (6) 4-6 keV, (7) 6-12 keV; (8) INTEGRAL/ISGRI hard X-ray band 18-63 keV; they are correlated with the light curves marked with dashed magenta lines. Right: Flux-flux diagram between the XMM/OM UV light curve in the $3-6 \mathrm{eV}$ band and the XMM/pn soft X-ray $0.2-0.5 \mathrm{keV}$ bands

correlation coefficient decreasing steadily with farther light curves. This behavior is exactly as expected in the case of two components, in this case the soft-excess and the "power-law" emission, whose respective contributions change with energy, one being dominant at low energy, while the other one is dominant at high energy. However, the same behavior extends down to the UV domain, with the correlation between the UV $3-6 \mathrm{eV}$ band and the soft X-ray $0.2-0.5 \mathrm{keV}$ band reaching $0.85\left(P<10^{-3} ;\right.$ Fig. 2 right). This is evidence that the blue-bump and soft-excess components are strongly linked. More precisely, it means that the blue-bump variability on time scales longer than $\sim 60$ ks has the same origin as the soft-excess variability. A single component, whose variability dominates that of other possible components at these energies, most probably covers the entire domain from the UV to soft X-rays.

\subsection{Broad-band continuum modeling}

We attempt to model the average emission of Mrk 509 from the UV to the hard X-rays with physically motivated emission components. The primary hard X-ray emission is often attributed to thermal Comptonization of the blue-bump photons in a hot corona [21]. We use here the xspec compps model [19] in a slab geometry. Hard X-ray emission is often observed to be reflected on distant, cold material, which we model with the xspec pexmon model [14]; pexmon is based on the standard pexrav model [11], but it includes some of the main emission lines, which are calculated in a self-consistent way. In order to explain the common variability of the blue-bump and of the soft-excess, we propose to add another thermal Comptonization component with a significantly colder temperature. As the required parameters are outside of the valid range for compps, we use instead the simpler nthcomp model [23]. In both Comptonization components, the soft photon 

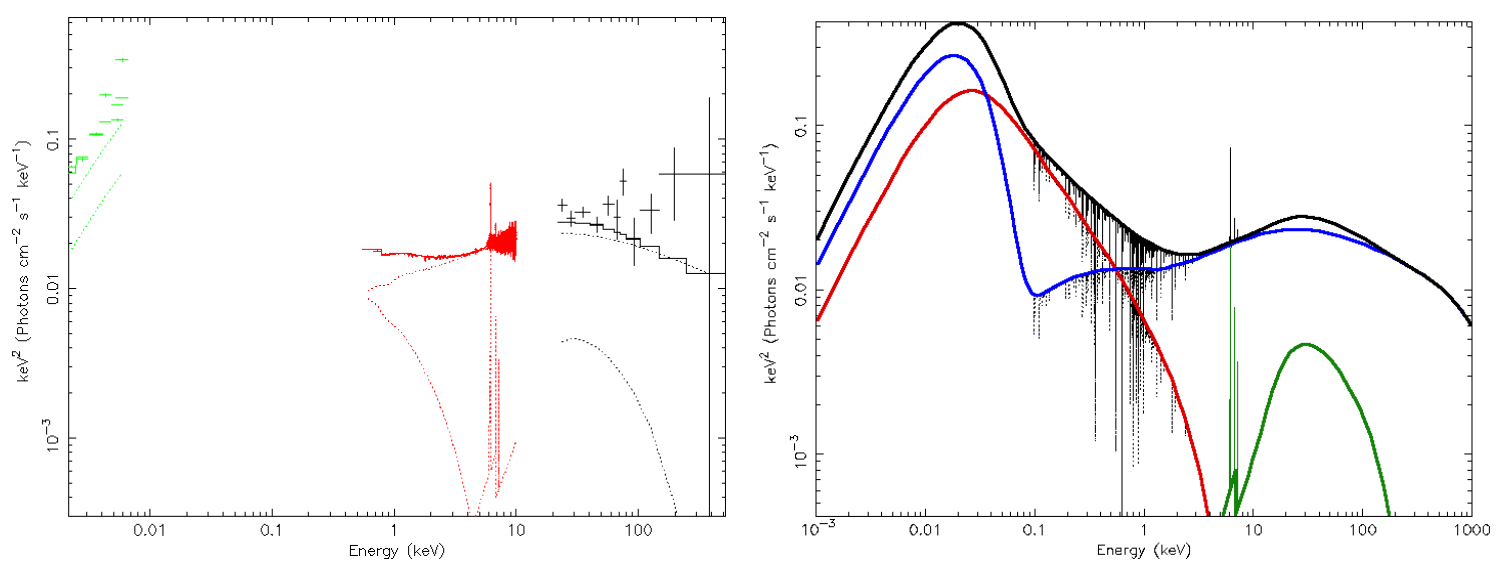

Figure 3: Broad-band modeling of the average Mrk 509 spectrum from $3 \mathrm{eV}$ up to $200 \mathrm{keV}$. The model consists of two thermal Comptonization components, a reflection model and neutral and ionized absorbers. Left: unfolded spectrum. The green, red and black points are the XMM/OM data, the XMM/pn data and the INTEGRAL/ISGRI respectively. Right: Fitted model without neutral hydrogen absorber to highlight the shape of the UV-soft-X emission. The blue curve is the thermal Comptonization component in a hot plasma; the red curve is the thermal Comptonization component in a warm plasma; the green curve is the reflection component. The black curve is total model; the ionized absorber component is visible around $1 \mathrm{keV}$.

component is modeled by an accretion disk component with a characteristic temperature of a few $\mathrm{eV}$, as determined in the UV. Neutral and ionized absorbers are also added to the fit. The absorber parameters have been fixed to the values determined in the XMM/RGS spectra [3]. Ionized absorbers with $\mathrm{N}_{\mathrm{H}} \sim 10^{21} \mathrm{~cm}^{-2}$ and $\xi \sim 80$ and $\sim 600$ have been found. Fig. 3 shows the best-fit results. The reflection fraction is found to be $R=0.34$. The very small formal uncertainty on $R$ ( $\sim 3 \%$ ) is due to its being firmly constrained by the iron emission lines. The hot-corona Comptonization component is characterized by a temperature of $\mathrm{k} T_{\mathrm{C}} \sim 470 \mathrm{keV}$ and an optical depth $\tau \simeq 0.025$. On the other hand, the plasma responsible for the soft-excess emission has a temperature of $\mathrm{k} T \sim 0.7 \mathrm{keV}$ and an optical depth of $\tau=16$. In our fits, all accretion disk photons cross either the hot or the cold plasma. Allowing a fraction of the photons to escape directly worsens the fit. As can be seen from Fig. 3 right, the blue-bump is dominated by photons escaping through the optically thin hot corona. The UV-soft-X correlation implies therefore that the variability of the warm-plasma emission dominates that of other components.

\section{Discussion}

\subsection{Origin of the soft-excess}

The extremely good correlation between the blue-bump and the soft-excess implies the existence of a single component covering both the blue-bump and the soft-excess energy domains and whose variability dominates that of possible underlying components. The lack of correlation with hard X-ray emission is a strong argument against blurred reflection. Nevertheless, the dominant component in the blue-bump emission is linked to the hard X-ray emission, as it consists, in our model, of the fraction of soft photons escaping without interaction from the hot corona. It seems 


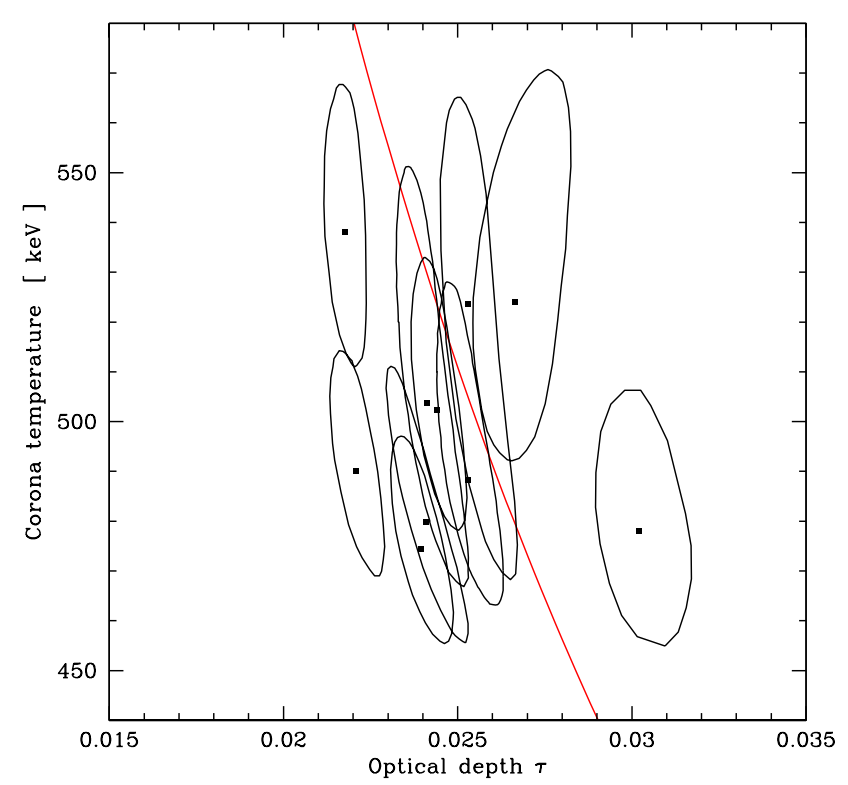

Figure 4: Corona temperature vs optical depth for the ten individual observations. The contours show the individual $1 \sigma$ uncertainties. The red curve is the theoretical curve for a plasma in radiative equilibrium over a passive disk in a slab geometry

however that the variability of this component in the blue-bump domain is negligible compared to that which is linked to soft-excess emission. The thermal Comptonization model in a warm, optically-thick plasma, explains however quite nicely the blue-bump and soft-excess linked variability. The plasma parameters $(\mathrm{k} T<1 \mathrm{keV}, \tau \sim 20)$ are quite comparable to the values found in other AGN, like NGC 5548 [10] or RE J1034+396 [13], strengthening the case for a Compton origin of the soft-excess in some objects at least.

\subsection{Physical conditions of the hot corona}

Thermal Comptonization in a hot corona is the standard interpretation for the hard X-ray emission in radio-quiet AGN. This model also provides a good explanation of this emission in the case of Mrk 509. However, the parameters are a bit at odds with those found in other objects, for which temperatures $\mathrm{k} T \sim 100 \mathrm{keV}$ and optical depths $\tau \sim 1$ are typically found [22]. The temperature is also incompatible with the low energy cutoff $(\sim 70 \mathrm{keV})$ found in [15]. The optical depth is also very low, with $\tau \simeq 0.025$. As a result, the vast majority of photons cross the corona without interaction. This result is very little model-dependent, as $\tau$ is mostly determined by the bluebump-to-hard-X-ray emission ratio. It must be pointed in addition that, thanks to the XMM/OM, HST/COS and FUSE data, the disk emission is much better constrained in the case of Mrk 509 than it has been the case for other AGN. Conversely, the corona temperature is quite high. In the case of a disk covered with a hot corona in a slab geometry, one can calculate the radiative equilibrium condition if one assumes that the disk is passive, i.e. that there is no internal heating [17]. Fig. 4 shows that the corona could indeed be in a situation of radiative equilibrium in Mrk 509, similarly to what is observed in NGC 7469 , although the temperature is lower in the latter. We can inter- 
pret this result as the evidence that most of the heating takes place directly within the hot corona. This radiative equilibrium condition is however not met in the other objects for which the corona parameters could be determined $[10,9]$.

\section{Conclusion}

The intensive broad-band campaign on Mrk 509 provides us with a unique window on the physical conditions in AGN. The inclusion of a detailed analysis of the UV emission is the biggest difference between this study and other similar works. The strong evidence for a thermal Comptonization origin of the soft-excess finds its origin in the UV-soft-X-ray correlation; the hot-plasma parameters are strongly constrained by the UV-to-hard-X-ray ratio. This kind of intensive campaign proves therefore to be very useful, and should ideally be reproduced in other similar objects and at different times to understand better the parameter space filled by type-1 AGN.

\section{References}

[1] Crummy, J., Fabian, A. C., Gallo, L., \& Ross, R. R. 2006, MNRAS, 365, 1067

[2] Dadina, M. 2008, $A \& A, 485,417$

[3] Detmers, R., et al. 2010, in preparation

[4] Fabian, A. C., Miniutti, G., Gallo, L., et al. 2004, MNRAS, 353, 1071

[5] Fabian, A. C., Zoghbi, A., Ross, R. R., et al. 2009, Nature, 459, 540

[6] Gierliński, M. \& Done, C. 2004, MNRAS, 349, L7

[7] Gierliński, M. \& Done, C. 2006, MNRAS, 371, L16

[8] Kaastra, J., et al. 2010, in preparation

[9] Lubiński, P., Zdziarski, A. A., Walter, R., Paltani, S., Beckmann, V., et al. 2010, MNRAS, 408, 1851

[10] Magdziarz, P., Blaes, O.M., Zdziarski, A.A., Johnson, W.N., \& Smith, D.A. 1998, MNRAS, 301, 179

[11] Magdziarz, P. \& Zdziarski, A. A. 1995, MNRAS, 273, 837

[12] Mehdipour, M., et al. 2010, submitted

[13] Middleton, M., Done, C., Ward, M., Gierliński, M., \& Schurch, N. 2009, MNRAS, 394, 250

[14] Nandra, K., O’Neill, P. M., George, I. M., \& Reeves, J. N. 2007, MNRAS, 382, 194

[15] Perola, G. C., Matt, G., Fiore, F., Grandi, P., Guainazzi, M., et al. 2000, A\&A, 358, 117

[16] Petrucci, P. O., Haardt, F., Maraschi, L., Grandi, P., Matt, G., et al. 2000, ApJ, 540, 131

[17] Petrucci, P. O., Maraschi, L., Haardt, F., \& Nandra, K. 2004, A\&A, 413, 477

[18] Ponti, G., et al. 2010, in preparation

[19] Poutanen, J. \& Svensson, R. 1996, ApJ, 470, 249

[20] Walter, R. \& Fink, H. H. 1993, A\&A, 274, 105

[21] Zdziarski, A. A., Johnson, W. N., \& Magdziarz, P. 1996, MNRAS, 283, 193

[22] Zdziarski, A. A., Poutanen, J., \& Johnson, W. N. 2000, ApJ, 542, 703

[23] Życki, P. T., Done, C., \& Smith, D. A. 1999, MNRAS, 309, 561 